\title{
Component Based Design of a Drug Delivery Capsule Robot
}

\author{
Marco Beccani ${ }^{a, *}$, Gregorio Aiello ${ }^{\mathrm{c}}$, Nikolaos Gkotsis ${ }^{\mathrm{a}}$, Hakan Tunc ${ }^{\mathrm{b}}$, Addisu Taddese ${ }^{\mathrm{a}, \mathrm{b}}$, Ekawahyu Susilo ${ }^{\mathrm{a}}$, Péter \\ Völgyesi $^{\mathrm{b}}$, Ákos Lédeczi ${ }^{\mathrm{b}}$, Elena De Momi ${ }^{\mathrm{c}}$, Pietro Valdastri $^{\mathrm{a}}$ \\ ${ }^{a}$ STORM Lab, Department of Mechanical Engineering, Vanderbilt University, Nashville, TN 37235-1592, USA \\ ${ }^{b}$ Institute for Software Integrated Systems, Department of Electrical Engineering and Computer Science, Vanderbilt University, Nashville, \\ TN 37212, USA \\ ${ }^{c}$ Department of Electronic, Information and Biomedical Engineering, Politecnico di Milano, Milano 20133, Italy
}

\begin{abstract}
Since the introduction of Wireless Capsule Endoscopy (WCE) researchers have started exploring the design space of Medical Capsule Robots (MCRs): embedded micro-systems that can operate autonomously within the human body and can diagnose, prevent, monitor, and cure diseases. Although the research in the area of MCRs is an active topic and has grown exponentially, current devices provide only limited functionalities because their design process is expensive and time consuming. To open this research field to a wider community and, at the same time, create better designs through advanced tool support, in our previous works we presented a design environment for the rapid development of MCRs. In this paper, this environment was adopted to design a Drug Delivery Capsule (DDC) based on a coil-magnetpiston mechanism. The force of the coil acting on the magnetic piston and the drug release profile were modeled and assessed on bench-top with a maximum relative error below $5 \%$. Then, in vivo trials were performed to validate the DDC functionality with a scheduled drug release profile for a 5 hours and 24 minutes procedure. The resulting design environment template is available open source for further development of drug delivery applications as well as to serve as guideline in prototyping novel MCRs addressing other clinical needs.
\end{abstract}

Keywords: Surgical robotics, medical cyber-physical systems, drug delivery, medical capsule robots, design environment, embedded micro-systems.

\section{Introduction}

The introduction in year 2000 of the PillCam by Given Imaging [1] showed that embedded micro-systems can be used to reach the more remote regions of the human Gastrointestinal (GI) tract. Since then, millions of patients have swallowed this self-contained wireless camera to get a non invasive diagnosis of diseases in the small intestine. Unfortunately, this approach is not effective for other districts of the GI tract, such as the colon and the stomach, where more deadly forms of cancer usually develop [2]. The main reason for this is related to the lack of advanced functionalities, such as active locomotion [3], advanced diagnosis and tissue manipulation [4], biopsy sampling [5], or drug delivery [6].

In recent years, the research community has addressed this issue by developing a number of robotic smart capsules, referred in this paper as Medical Capsule Robot (MCR). MCRs typically enter the human body through natural orifices or small incisions and can perform endoscopy and surgery by interacting with the surrounding environment with sensors and/or actuators.

\footnotetext{
*Corresponding author, E-mail address: marco.beccani@vanderbilt.edu
}

The design process of MCRs however is challenging because it has to address severe cross-cutting constraints such as size (to gain non invasive access, MCR diameter is limited to about $1 \mathrm{~cm}$ ), power consumption (limited space for battery is available onboard), and fail safe operation (MCR operates deep inside the human body). Therefore, MCR design and development requires significant skills and efforts in embedded systems, miniaturized electronics, packaging, debugging, and mechanical miniaturization of the device. Custom circuit boards and mechanical enclosures have to be engineered, which makes MCR development an expensive and time consuming process that is only accessible to a limited number of groups in the research community [7].

Because many of the MCRs developed in the past share equivalent hardware and software components such as specific sensors, data processing, actuation, and wireless communication, we believe that it is indeed possible to systematize the design of MCRs. This would lower the barrier of entry to this field to research groups that do not have the resources to develop MCRs from the ground up.

In our previous works, we presented our contributions towards the creation of an environment for the rapid design and development of MCRs [7, 8, 9]. The basic elements of the integrated design environment are shown in Fig. 1. 


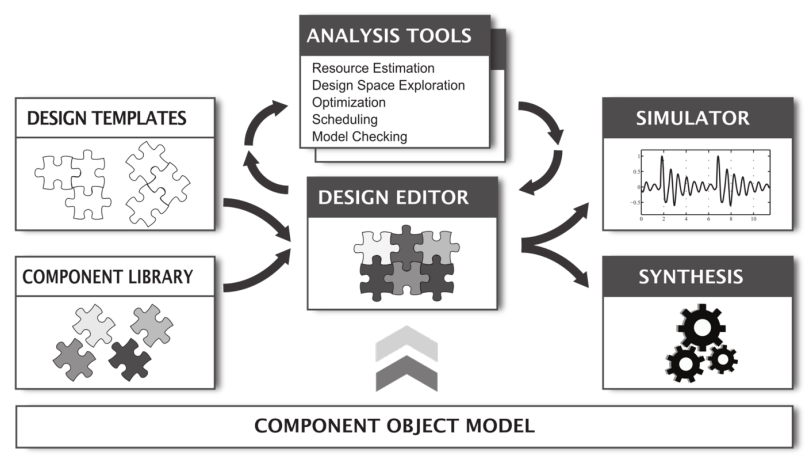

Figure 1: The elements of the integrated design environment for MCR.

At the time of writing, the design environment includes a library of hardware modules built up from our experience with existing capsule designs [10]. The hardware components include fundamental elements of MCR design such as sensors (inertial sensors, encoders, vital sign sensors), actuators (DC motors, coils, illumination, servo motors, stepper motors), batteries and voltage regulators, wireless transceivers working at different carrier frequencies, and microcontrollers (MCUs), which serve as building blocks for the system. A flexible backbone enables plug-n-play connectivity of up to six hardware modules. Each of the hardware modules is coupled with a software component and integrated in an open-source web environment available at pillforge.github.io for the developer to implement specific MCR applications.

In this work, we present how the design environment can be adopted to develop a Drug Delivery Capsule (DDC) based on a coil-magnet-piston mechanism for controlled deployment of drug doses over time. As a result of this work, we have created a design template for the DDC. This template aims to provide MCR researchers a solution that shortens the development time of new DDCs based on the same or a similar actuation principle. Template parameters such as the drug viscosity and deployment schedules can be adjusted according the specifics of the application. The force exerted by the coil-magnetpiston mechanism and the drug release profile over time have been modeled and characterized experimentally. Beyond the specific application of controlled drug delivery, this work also illustrates how the open-source design environment we have developed can be used to rapidly design an MCR.

This paper is organized as follows: Section 2 briefly presents the state of the art for drug delivery MCRs, Section 3 illustrates the mechanical, electrical, and embedded firmware and software design considerations for the developed MCR. This section also describes the design environment and the design template that have been created for the MCR. Section 4 presents the experimental assessment on bench and in vivo results. Section 6 discusses conclusions and future research directions.

\section{Clinical Motivation for Drug Delivery Systems}

The formulation of drugs that allow for reproducible absorption profiles for targeting specific areas of the GI tract represents a major bottleneck in current development of orally administered medications [11]. This is mainly due to unpredictable gastric transit time and physiochemical characteristics of the GI tract that differ from patient to patient [12].

The availability of a controllable Drug Delivery Systems (DDS) may reduce toxicity associated with systemic administration and prevent the formation of antibodies to drugs, particularly in the case of colon cancer or inflammatory bowel disease therapies [13]. These are two extremely common diseases, with colorectal cancer being the second leading cause of cancer-related deaths in USA [14], while Crohns disease and ulcerative colitis - both inflammatory bowel diseases - being diagnosed in 201 and 238 per 100,000 people in the USA, respectively.

Controllable MCRs targeting drug release have been developed for therapeutic treatment of diseases in the GI tract $[6,15]$ as well as for drug absorption studies [16]. Although some devices are already commercially available, such as the IntelliCap [17], the InteliSite [18], or the Enterion capsule [19], they cannot implement an intelligent release algorithm due to the lack of real-time localization and active position control. Several MCRs have been proposed to address these limitations. Yim et al. [20] developed a magnetic locomotion system that can collapse under magnetic guidance and is able to deploy a liquid drug. Kim et al. [21] proposed a three-axis Helmholtz coil capsule which moves with a rotational motion and deploys a drug. Woods et al. [22] integrated a holding mechanism inside the capsule to resist to peristalsis and a needle for local drug deployment. The MAARS capsule, presented in [23], is composed of magnetic semi-hard modules and deploys a single shot of drug upon a demagnetization process. Yo et al. [24] recently proposed an MCR where the drug release can be triggered through a reed switch. Finally, Hafezi et al. [25] developed an ingestible sensor that allows patients, families, and physicians to monitor prescription compliance and drug-adherence patterns in real time.

While each of these capsules taken alone solves a specific problem, none of them offers a complete solution to achieve an intelligent release of a drug on a specific target. In order to do that, multiple functionalities need to be combined and several design iterations need to be tested. Thanks to the modular approach adopted in this work, we have done a first step in that direction by implementing a controllable mechanism for drug release and an intelligent scheduler. These components take only part of the available slots on the flexible circuit backbone, leaving space for adding other functional modules, such as localization and/or active position control. 


\section{Design Consideration}

\subsection{Principle of Operation - Mechanism Consideration}

Referring to Fig. 2, where the principle of operation of the MCR is presented, the DDC mechanism consists of a drug chamber, two coils, and the magnetic piston. The drug chamber $(\mathrm{d}=7.94 \mathrm{~mm}, \mathrm{l}=6.35 \mathrm{~mm}$, volume $=$ $314.42 \mathrm{~mm}^{3}$ ) is hosted in a cylindrical enclosure together with an axially magnetized cylindrical permanent magnet acting as a piston (D54-N52, K\&J Magnetics, USA). The DDC shell and the drug chamber were prototyped with an Objet30 3D printer (Stratasys, USA) with Vero white material. The clearance between the outer diameter of the magnetic piston and inner diameter of the chamber was 0.4 $\mathrm{mm}$. This value guaranteed a low friction with the magnet, resulting in no leakage of the drug and the actuating mechanism. The distal collar edge of the chamber has twelve circular holes (each with a radius of $r_{h}=0.8 \mathrm{~mm}$ ) from where the drug is released into the environment. The number of holes and their radius have been chosen such that the drug is deployed uniformly without being affected by capillarity. In case the application requires a drug with a different viscosity, the number of holes as well as their dimension can be adjusted. The combination of proper drug viscosity and aperture size prevents the drug from leaking for any possible orientation of the capsule. Bench trials showed that the proposed design is able to deploy solutions with a viscosity up to $1000 \mathrm{cP}$, which is higher than water $(0.89 \mathrm{cP})$, blood (between 3 and $4 \mathrm{cP}$ ) and air $(0.018 \mathrm{cP})$.

To actuate the magnetic piston, two coils are mounted at both sides of the drug chamber such that, when they are activated, the current $I$ induces two static magnetic fields aligned along the same axis, having the same magnitude, but opposite directions.

The force $F(d z)$ induced by the two coils on the magnetic piston can be expressed as a function of the relative position $d z$ of the piston with respect to the two coils. The trend of $F(d z)$ was modeled with COMSOL multiphisics (COMSOL Multiphysics, Stockholm, Sweden) and experimentally verified as discussed in Section 4. Considering the surface of the magnetic piston $S$, we can derive the pressure $\mathrm{P}(\mathrm{dz})$ in the chamber as a function of the magnetic piston position $d z$ :

$$
P(d z)=\frac{F(d z)}{S} .
$$

Using the Hagen-Poiseuille's equation [26], we can then express the fluid flow $\dot{V}$ as a function of the pressure assuming that the radius of the holes is smaller than their length, the drug is a Newtonian fluid, and the motion of the resulting drug flow across the holes is laminar. Under these assumptions, we have:

$$
\dot{V}=n \cdot \frac{P(d z)}{R_{h}}
$$

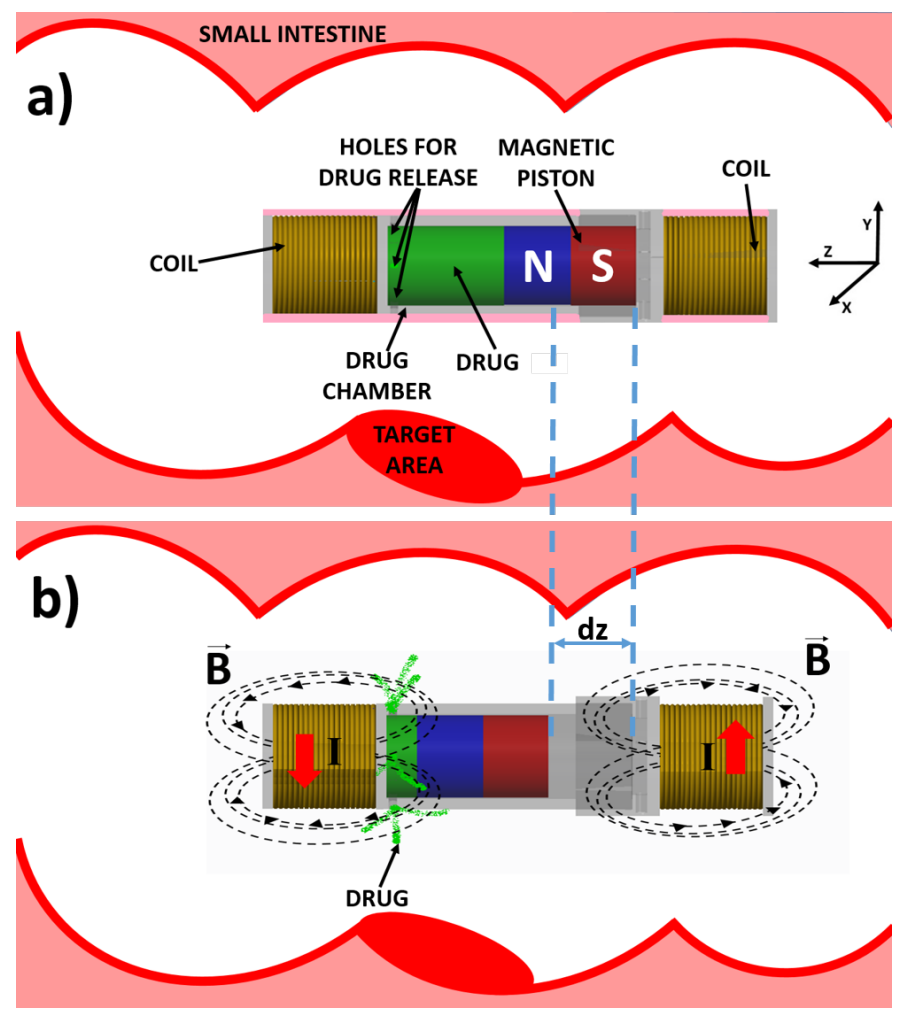

Figure 2: Principle of operation of the DDC (a). When the coils are activated (b) the magnetic piston deploys the drug outside of the drug chamber.

where $R_{h}=\frac{8 \rho l}{\pi r^{4}}$ is the hydraulic resistance, $\rho$ is the dynamic viscosity of the drug, while $l, r$ and $n$ are the length, the radius and the number of holes in the chamber, respectively.

From the flux $\dot{V}$, it is possible to derive the quantity of drug released $M$ as

$$
M=\dot{V} \cdot t_{a c t} .
$$

with $t_{a c t}$ being the time period when the coil is active.

Coil Dimensioning. The magnetic force acting on the piston is proportional to the magnetic field $\mathrm{B}$ generated by the coils as

$$
F=\frac{B^{2} \cdot A}{\mu}
$$

where $\mathrm{F}$ is the force, $\mathrm{B}$ is the magnetic field, $\mathrm{A}$ is the section of the coil, and $\mu$ is the magnetic permeability of the medium (i.e., air, therefore $\mu=\mu_{0} \mu_{r} \simeq \mu_{0}$ ). Since $\mathrm{B}$ is a proportional to the current $I$, the coil dimensions, and the number of turns, we need to optimize the three quantities to achieve enough force to push the magnet along the chamber against the drug.

On the other hand, the amount of current in the coil is limited by the power dissipated by its internal resistance as heat. For safety reasons, the capsule temperature should not increase by more than $1^{\circ} \mathrm{C}$ [27]. Since the coils are mounted in a parallel configuration, their total resistance 
is $R_{T C}=\frac{1}{2} R_{C}$ with $R_{C}$ resistance of a single coil. The maximum current that can flow through the coil wire without causing a $1^{\circ} \mathrm{C}$ increase is equal to $800 \mathrm{~mA}$. Adopting a value of $700 \mathrm{~mA}$ for the current, and considering that the coil is supplied at $3.3 \mathrm{~V}$, we have:

$$
R_{T C}=\frac{V}{I}=\frac{3.3}{0.7}=4.71 \Omega
$$

thus $R_{C}=9.42 \Omega$ for each coil. Since the wire resistivity is $\rho=17.421 \cdot 10^{-9} \Omega m$ and its is section $S_{w}=1.5386$. $10^{-8} \mathrm{~m}^{2}$, the length of the wire $L$ results in:

$$
L=\frac{R \cdot S_{w}}{\rho}=8.32 \mathrm{~m} .
$$

Considering that the wire is wrapped around a circumference of $C_{i n}=0.0157 \mathrm{~m}$, the number of turns $N$ results in

$$
N=\frac{L}{C_{\text {in }}}=\frac{8.32}{0.0157}=530.394 \approx 530 \text { turns }
$$

Once wrapped, the coil results in a diameter of $10 \mathrm{~mm}$, a length of $18 \mathrm{~mm}$, and an inductance of $1.5 \mathrm{mH}$.

\subsection{Hardware Architecture}

The hardware architecture for the presented MCR consists of two separate systems: a base station and the DDC itself. The base station [7] was used to exchange wireless data between the MCR and the user interface. In this particular application, the user might desire to change the delivered therapy schedule after the capsule has been ingested, thus the base station is the only way to interact with the DDC.

The DDC electronics consists of three embedded modules: the MCU, a $433 \mathrm{MHz}$ transceiver, and the powering and battery monitoring module. The modules, are mounted on our flexible circuit. These components are shown in Fig.3 before the flexible circuit is folded to fit a capsule-shaped shell. The remaining three empty slots on the flexible circuit can be used in the future to add other functional modules. The MCU module is based on a MSP430 (MSP430F5528, Texas Instruments, USA), which communicates via Serial Peripheral Interface (SPI) with the $433 \mathrm{MHz}$ transceiver (CC1101, Texas Instruments, USA) module. The driver for the coil actuation circuitry consists of a power p-channel MOSFET (STR2P3LLH6, ST Microelectronics, SUI), which has been mounted directly on top of the flexible circuit. The two coils are connected to the drain of the MOSFET. This module can provide up to $800 \mathrm{~mA}$. The MOSFET gate is driven by a MCU digital output with a Pulse Width Modulation (PWM) signal. Finally, power to the DDC is provided by a $30 \mathrm{~mA} / \mathrm{h} \mathrm{LiPo}$ rechargeable battery (LP-FR30, Plantraco LTD, USA). Accordingly to the drug scheduler, the DDC deploys the drug only at specific times. The battery lifetime therefore has to be optimized to minimize the MCR power consumption. This is achieved by running the MCU in low power

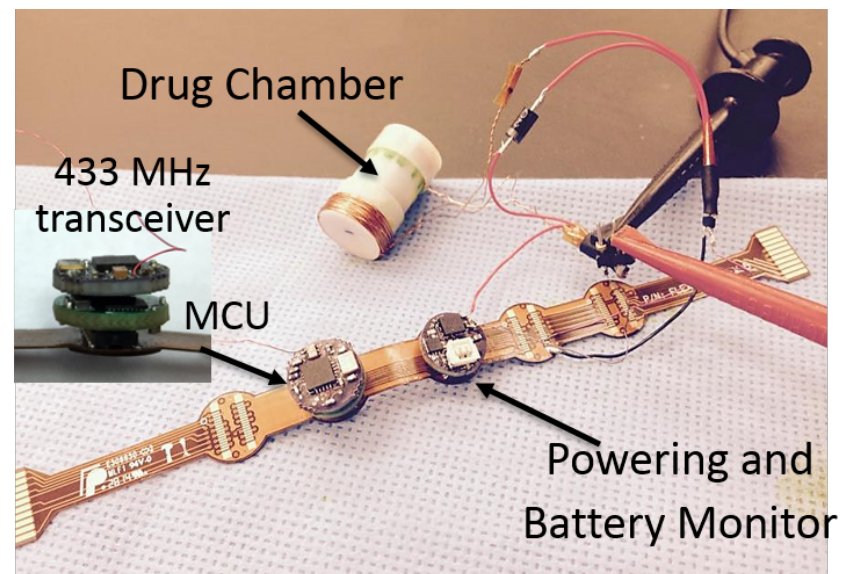

Figure 3: Picture of the embedded electronics of the DDC before folding the flexible circuit. The lateral connectors at the opposite sides of the flexible circuit are used for programming and debugging the modules and are removed before integration into the capsule shell.

mode, and activating its peripheral interfaces (e.g., wireless communication, actuation) on demand. Table 1 shows the power consumption of the DDC embedded electronics during different operating modes.

All the documentation related to the base station as well to the other miniature hardware modules is available at pillforge.github.io.

\subsection{Design Environment \& Scheduler}

Developing an embedded application can be intimidating for novice users. Applications are usually coded with low-level languages like $\mathrm{C}$, which presents a barrier for researchers whose expertise lies in the applications of MCR. In addition, setting up tool chains and the necessary software libraries for a specific target device is usually cumbersome. To alleviate these hardships, we have developed a web-based visual software design environment [7].

The design environment provides a higher level of abstraction for applications, making it easier for developers to reason about their software. A user can compile his/her application through the design environment and download its binaries through the web interface. Since the compilation is processed on the server side, the user does not need to install any additional tools.

We applied a proven model-based methodology [28] to the MCR domain. This method allowed us to visually represent software models specific to MCR and to generate the corresponding software artifacts. The compositional features of model-based development's provide additional abstractions that can encapsulate the underlying complexities. The design environment was implemented using the Web-based Generic Modeling Environment (WebGME), which is a modeling tool used to design domain-specific visual modeling languages [29].

We have chosen TinyOS as the core operating system for the MCR. TinyOS is an event-driven, operating system commonly used in small resource constrained 


\begin{tabular}{|l|r|r|r|r|}
\hline Module & Sleep Mode & Idle Mode & Tx or Rx Mode & Drug Deployment \\
\hline MCU & $100 \mathrm{nA}$ & $1.8 \mathrm{~mA}$ & $18.3 \mathrm{~mA}$ & $32.5 \mathrm{~mA}$ \\
\hline 433MHZ transceiver & $200 \mathrm{nA}$ & $8.4 \mathrm{~mA}$ & $14.7 \mathrm{~mA}$ & $14.7 \mathrm{~mA}$ \\
\hline Power Board & $8 \mu \mathrm{A}$ & $0.8 \mathrm{~mA}$ & $0.8 \mathrm{~mA}$ & $0.8 \mathrm{~mA}$ \\
\hline Coil & $0 \mathrm{~A}$ & $0 \mathrm{~A}$ & $0 \mathrm{~A}$ & $700 \mathrm{~mA}$ \\
\hline Total & $8.3 \mu \mathrm{A}$ & $11 \mathrm{~mA}$ & $33.8 \mathrm{~mA}$ & $748 \mathrm{~mA}$ \\
\hline
\end{tabular}

Table 1: Power consumption of the embedded modules during the different modalities of operation of the DDC: sleep, idle, wireless transmission or reception, deployment of the drug.

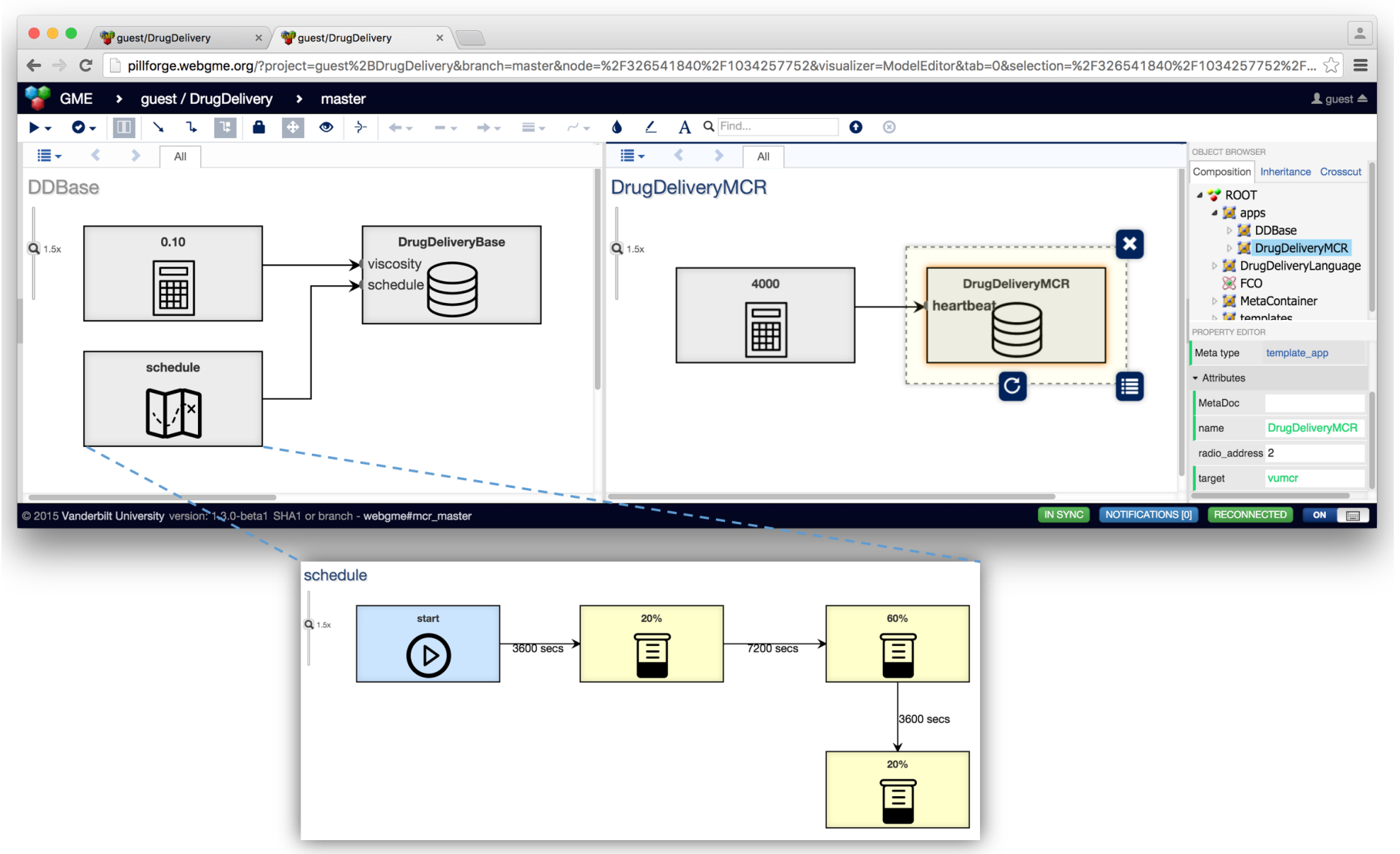

Figure 4: The design environment showing Base and MCR apps with the highlight of the schedule.

devices [30]. Its component based paradigm lends itself naturally to the model-based methodology of our design environment. Hence, a modeling language was created using WebGME to represent TinyOS language constructs. A one to one mapping of TinyOS software components to WebGME's notion of components allows users to create applications and visualize the interconnection and structure of the constituent software components. However, this still requires users to implement their application logic in TinyOS, which has a steep learning curve.

Instead, a higher level of abstraction is needed to connect the users' intentions to actual TinyOS components. To this end, we have added blocks to our previous [8] meta language definition of TinyOS to support a higher level of abstraction. Blocks are WebGME components that expose customizable inputs, outputs, and parameters to users. They also abstract data types and allow users to visu- ally connect data to the apps without writing any textual code.

In this work, we have created two template apps that can be used in the DDS, which is composed of the base station and the MCR. The corresponding software models can be seen in figure 4 . For the base station, the schedule and viscosity blocks are connected to a template drug delivery base app, while the heartbeat rate value block is connected to a template drug delivery MCR app's port. MCR sends a status message periodically to the base station. The status messages used for the communication protocol between MCR and the base station as well as to signal to the base station that the MCR is still alive (hence the name heartbeat). Once the communication is established between the devices, the remaining percentage of drug is used as the payload in the status messages. The heartbeat rate value determines the frequency of messages 


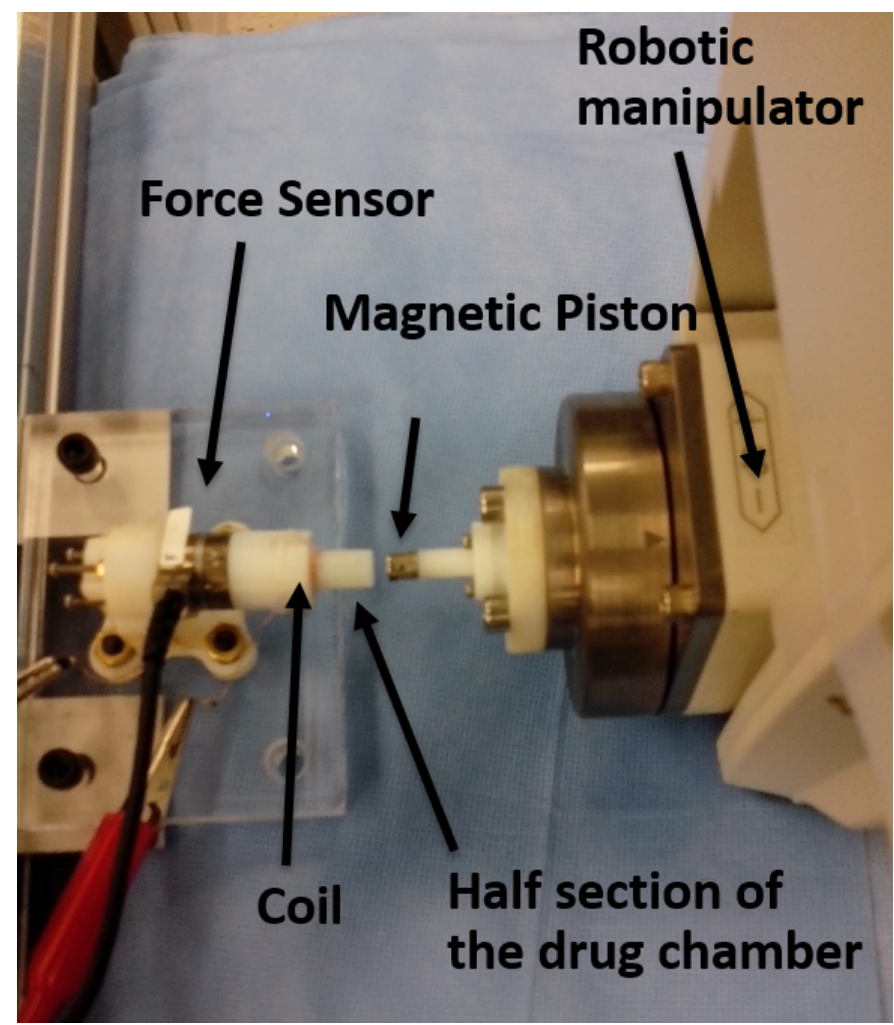

Figure 5: The experimental setup to assess the force $\mathrm{F}(\mathrm{dz})$ inside the drug chamber while the coils are active.

send from the MCR. In addition, the template apps have attributes to set the radio address for wireless communication.

The internal structure of the schedule block can be seen in Fig. 4. The blue object represents the beginning of a schedule, the green elements represents the amount of drug to be released. Blue and green elements are connected with time connections. The schedule block dictates what percentage of drug should be released in the specified time intervals.

Once the schedule and the parameters are set, a user can compile the application by running the compiler plugin via the arrow button located on top left of the design environment. The generator traverses the input data and feeds it to the template app. The app gets compiled on the server side, the resulting binaries, in turn, can be downloaded by the user. The software is available at https://github.com/pillforge/drug.delivery.

\section{Device experimental assessment}

Experimental trials aimed to assess and characterize the DDC functionality were performed. The interaction force exerted from the two coils on the magnetic piston was modeled and experimentally validated. We also verified that the DDC, once programmed with a scheduler, delivered the amount of drug expected from the release profile described in Section 3.1. Finally, the reliability and

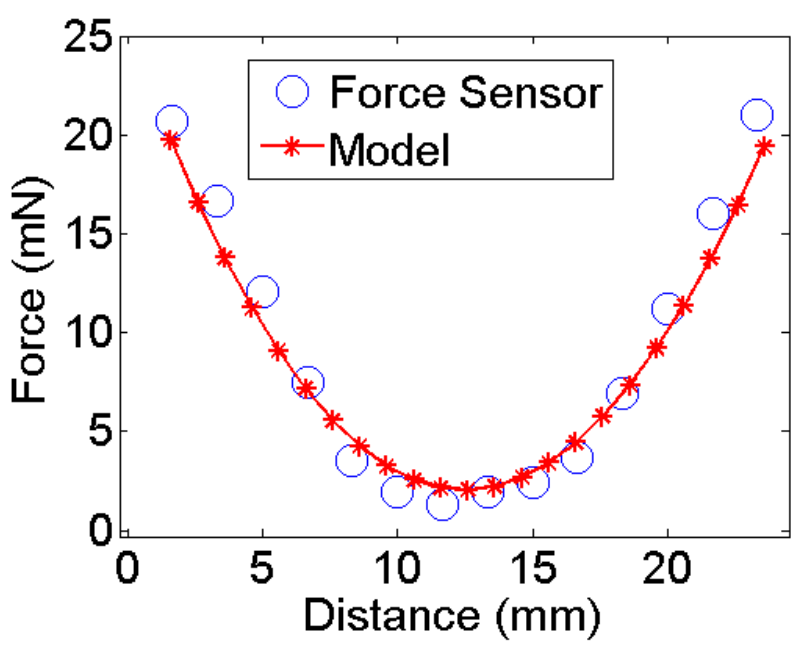

Figure 6: Experimental data measured from the load cell compared to the finite element analysis simulation.

functionality of the device were tested in an in vivo trials on a canine model.

\subsection{Validation of the interaction force between the coils and the magnet}

The interaction force between the coils and the magnet has been modeled using finite element analysis (COMSOL) by assuming the two coils as Helmholtz coils with the following geometrical and electrical parameters: number of turns $N=531$, resistivity of the wire $\rho=17.421$. $10^{-9} \Omega \mathrm{m}^{2}$, radius of the coil $r_{c}=2.5 \mathrm{~mm}$, current flowing in each coil $I_{c}=350 \mathrm{~mA}$, diameter of the wire $d_{w}=$ $0.2 \mathrm{~mm}$, and length of the coil $L=1 \mathrm{~cm}$. The motion of the magnetic piston occurs along the $\mathrm{z}$ direction of the DDC, thus we extracted from the simulation the numerical values for the interacting force $\mathrm{F}(\mathrm{dz})$ as a function of the piston position into the drug chamber. The same quantity was then experimentally measured with the setup presented in Fig. 5. A rapid prototyping part (Objet 30, Objet Geometries Ltd, USA) was designed to mount a 3-axis force sensor (NANO17, ATI Industrial Automation, USA) with the coils and the drug chamber. Opposite to the chamber, the magnetic piston was mounted at the end effector of a six-degree-of-freedom robotic manipulator (RV6SDL, Mitsubishi Corp., Japan). While the coils were active, the magnetic piston was moved inside the chamber for its entire length, while the resulting force was collected using a Universal (Serial Bus (USB) data acquisition board (NI-PCI 6224, National Instruments, USA) at a sampling rate of $100 \mathrm{~Hz}$.

The graph of the modeled force and the experimental data are represented in Fig. 6. The plot shows a parabolic and symmetric trend for the force, which reaches its minimum exactly at the center of the chamber, halfway between the coils. The relative error between the finite element simulation and the force sensor data was $3.4 \%(\sigma=$ $\pm 2.5 m N)$. 
In order to validate the effect of gravity on the drug delivery mechanism, the trials were performed with the capsule assuming different orientations. Since the contribute of gravitational force is negligible compared to the magnetic force generated by the coil, the mechanism was able to withstand it regardless of the capsule orientation.

\subsection{Validation of the drug released}

This trail aimed to verify the ability of the DDC to release a scheduled quantity of drug with time. In Sec. 3.1 we derived expressed the quantity of drug released $M$ as a function of the flux $\mathrm{V}$ and the time of activation of the coils. This relationship was verified on bench with in this experimental setup.

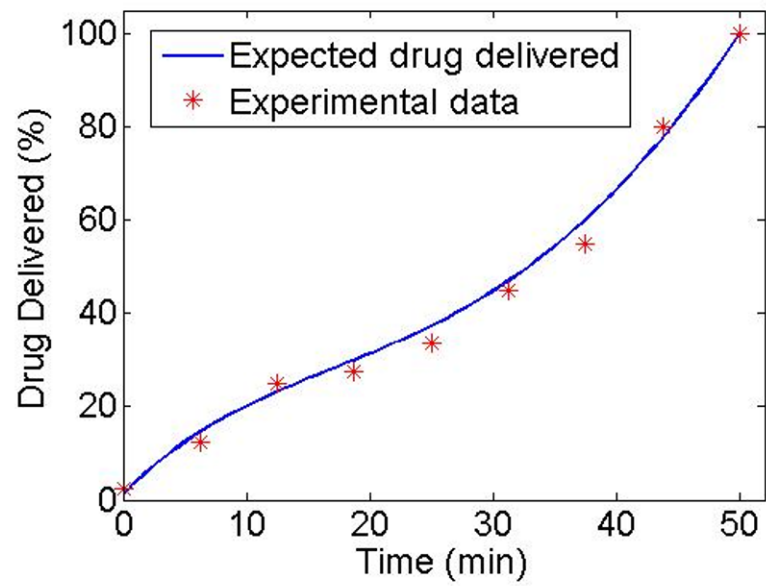

(a)

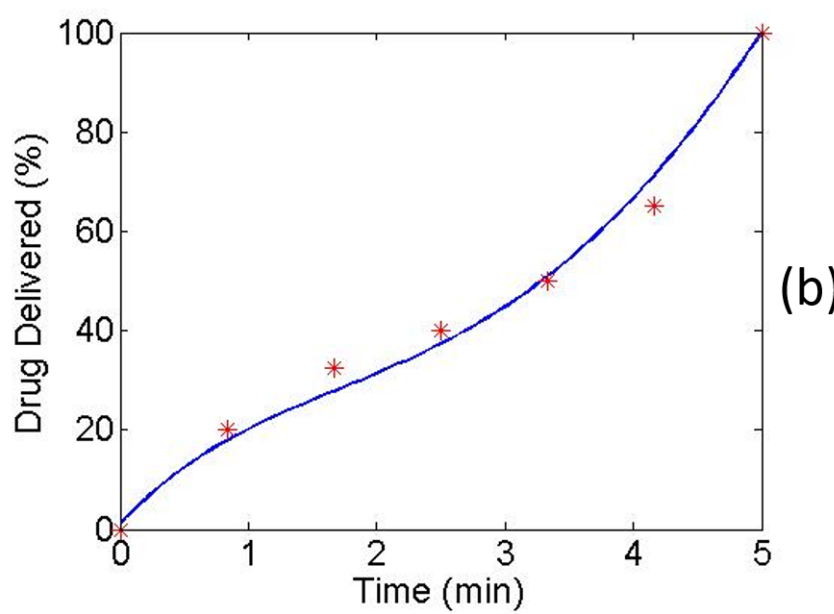

Figure 7: Predicted drug delivery profile (in blue) compared with the experimental data (in red) for one of the trial.

The capsule drug chamber was filled with a solution of $40 \%$ water and $60 \%$ lubricant, resulting in a dynamic viscosity of $0.032 \mathrm{~Pa} \cdot \mathrm{sec}$ and with a solution of $50 \%$ water and $50 \%$ lubricant, resulting in a dynamic viscosity of $0.024 \mathrm{~Pa} \cdot \mathrm{sec}$. The MCU was programmed to deliver a scheduled quantity of solution every 7 minutes and thirty seconds accordingly to the scheduler profile. Every time that the drug release was triggered, the MCR was placed on a digital scale (American Weigh Scales, Inc, USA, resolution $0.01 \pm 0.005 \mathrm{~g}$ ) and its weight recorded. The quantity of solution released with time was thus derived from the capsule initial weight, when the drug chamber was completely filled up. Ten different experimental trials were then performed. The results of one of the trial for the two different viscosity is shown in Fig. 7, where the expected drug deployment profile (in blue) is compared to the actual experimental profile data (in red). Repeatability tests for delivering $10 \%$ of the drug loaded into the chamber resulted in an average drug aliquot of $72 \mu \mathrm{L}$ with a standard deviation of $0.28 \mu L$.

A good matching of the experimental data with the expected drug release profile was observed. Overall, the relative error for the quantity of drug released vs. its expectation is $2.4 \% \pm 1.8 \%$. Current consumption of the device matched the values reported in Table 1 with a relative error equal to $3 \%$. In particular, the current in the coil was $695 \mathrm{~mA}$.

\subsection{In Vivo Trial}

The in vivo trial was performed at Vanderbilt University under protocol M/14/014, that included other devices and experiments, using a $25 \mathrm{~kg}$ female dog under general anesthesia. The DDC drug chamber was filled with a solution of $60 \%$ water and $40 \%$ lubricant with a green dye, resulting in a dynamic viscosity of $0.05 \mathrm{~Pa} \cdot \mathrm{sec}$. The capsule was then programmed to schedule a drug delivery of $7 \mu \mathrm{L}$ every $15 \mathrm{~s}$ until all the drug in the chamber was completely deployed for a total of 6 doses.

The DDC was inserted from the anus into the colon using a standard colonscope and left in place for the amount of time needed for the trial. The same colonscope was used to observe the release of the drug under insufflation of the colon. Figure 8 shows the sequence of the DDC deploying a scheduled drug doses: the drug release is first triggered (A), then the drug starts to flow outside of the chamber (B) and reaches the colon tissue (C).

The current consumption of the DDC during the trial was estimated as in [31] using the following average current consumptions:

$$
\bar{I}=\frac{\sum_{i} I_{i} T_{i}}{\sum_{i} T_{i}},
$$

where $\mathrm{I}_{i}$ and $\mathrm{T}_{i}$ are the current consumption from Tab. 1 and the time interval related to a single action performed by the DDC, respectively. For our particular application $\bar{I}$ resulted equal to $28 \mathrm{~mA}$. Adopting a battery with a capacity of $250 \mathrm{mAh}$, we can expect a maximum lifetime of $T=\frac{250}{28} \approx 8 h$ and $50 \mathrm{~min}$. The in vivo trial showed that the device was able to operate for at least 5 hours and 24 minutes. After that, all the drug was deployed and the trial was considered as concluded. In case that a longer operating time were needed, battery lifetime can be further extended by increasing the MCU sleep intervals. Crohn's disease and ulcerative colitis are inflammatory small bowel 

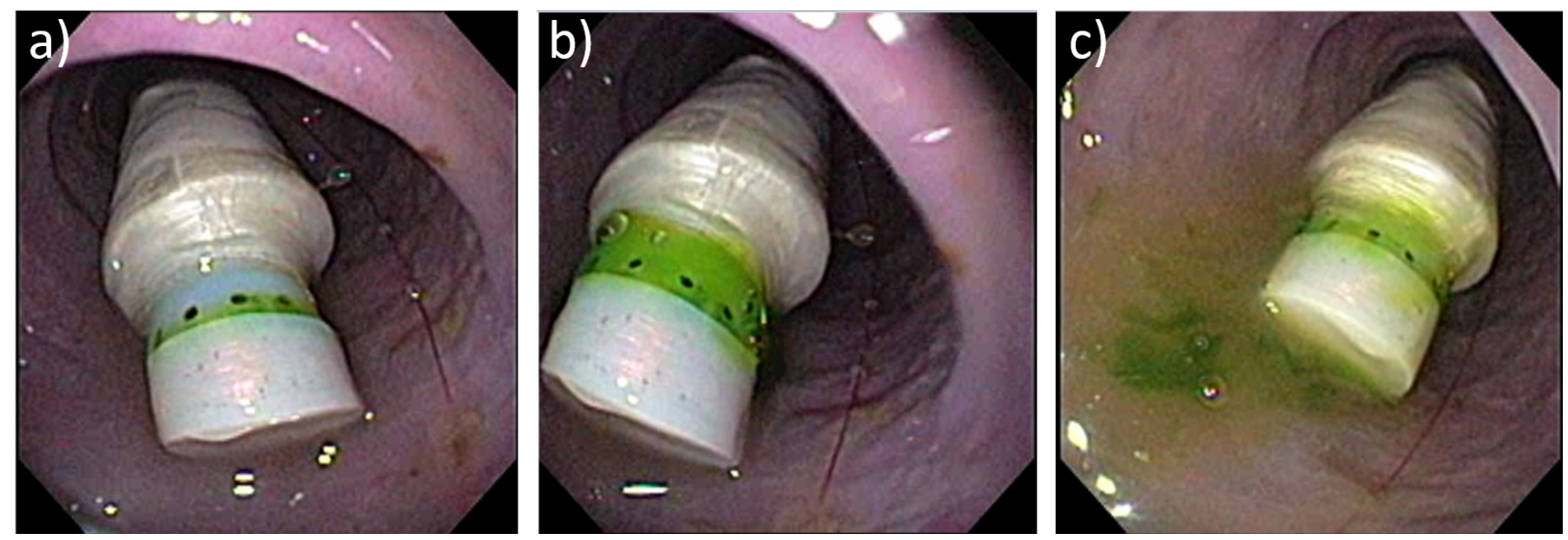

Figure 8: A sequence from the in vivo trials. The drug deployment is triggered (A), the drug release starts (B), and the dose reaches the colon tissue $(\mathrm{C})$.

diseases that are typically treated by releasing controlled quantities of drugs over a period of time. While the typical transit time for $50 \%$ of food in the small bowel is up to three hours, human clinical trials in this GI tract for similar MCR, (e.g. the Intellicap) have shown an average small bowel transit of up to 5 hours. The actual battery lifetime guarantees enough time for the device to operate while traveling across the small bowel. However, since the delivery time and the quantity of drug deployed can be adjusted with the scheduler, different diseases can be targeted. The purpose of the animal trial was to qualitatively assess the functionality of the DDC in a clinical scenario. The main limitation of the trial compared to a clinical scenario was that the device was not swallowed, because of the protocol trial time. The goal of the trials was to assess if the device was able to properly operate inside a constrained environment, being able to respond to wireless commands, and reliably actuate the mechanism. For this purpose, we first tried the device without insufflation. After the scheduled time of drug delivery, we insufflated the colon and we checked proper deployment of the drug. At this point, we run a trial with insufflation to capture a video footage.

\section{Discussion}

As discussed in [7], the main purpose of our modular design environment is to accelerate the time-to-prototype for the early validation of design hypothesis in the field of MCRs. The time-to-prototype depends principally on the complexity of the hardware and software of the MCR as well as the skill-set and experience of the developer.

In particular, hardware complexity consists of circuit design, assembly of miniature components, and testing. While a custom approach requires the developer to go through these iterative steps until the desired level of minia- turization and performance is achieved, our modular design environment provides the user with miniaturized hardware modules and a flexible circuit for easier connectivity. The same degree of miniaturized connectivity can be achieved in custom designs with flexible PCB cables or hybrid PCB solutions, but they are known to be expensive for the low volumes that are typical for research and proof-of-concept validation [32].

Software complexity consists of writing efficient embedded $\mathrm{C}$ code to implement the various functions of an MCR and to communicate with external components such as sensors, actuators and wireless base stations. Instead, our modular design environment provides the developer with a set of software components, which can be customized according to the requirements of the application. Ultimately, the experience and skill-set of the developers involved determines how efficiently the described hardware and software complexities are managed. Exact quantitative benefits of our component based design further depend on the specifics of the application, however, to give to the reader an idea of the time and cost outcomes, the swimming capsule MCR described in [7], has been developed and assembled in less than two months with an overall estimated cost of 15,000 USD (including materials and support for personnel) compared to nine months and an estimated cost of 50,000 USD for the MCR presented in [33].

It is worth mentioning that the hardware platform we propose, being a system made of modules, is not optimized for any particular application. Thus, MCRs built with our platform are generally larger than their custom counterparts, as shown in Fig. 3 compared to Fig. 9.

\section{Conclusions}

In this work, we presented a component based design of a DDC robot based on a coil-magnet-piston mechanism. This device aims to provide a controlled deployment of 

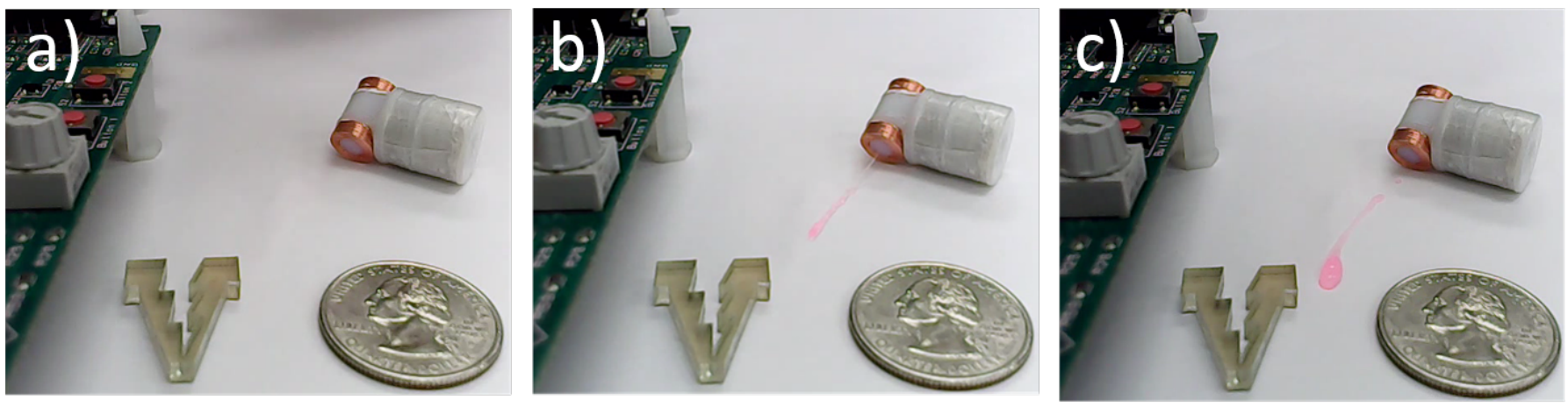

Figure 9: a) A picture of the final DDC prototype, where the embedded electronics was developed via custom design. The device is 25 mm long for a diameter of $16 \mathrm{~mm}$. b) The drug stars to be released and c) is completely deployed.

drug doses over time with a scheduler. The drug viscosity and deployment time can be set in the application template for the design environment according to the desired drug release profile. The magnetic force as well as the drug release profile have been modeled and then experimentally verified. The models for the force and the released drug profile showed relative errors below $5 \%$ when compared to the experimental data. The functionality of the DDC was also successfully assessed in a clinical scenario via an in vivo trial.

In this work, we validated the hypothesis that a coilmagnet-piston mechanism is a viable solution for a scheduled drug release. However, the DDC that we developed by assembling the preexisting modules from our library resulted in a size that is impossible to swallow. Therefore, once we were confident with the coil-magnet-piston mechanism operation, we scaled down our design to an ingestible size by designing a custom flexible circuit. The DDC represented in Fig. 9 is $16 \mathrm{~mm}$ in diameter and $25 \mathrm{~mm}$ in length with a drug reservoir of $201.06 \mathrm{~mm}^{3}$. This capsule contains a scaled version of the coil-magnet-piston mechanism described in this paper and it is capable of deploying drugs with viscosities up to $700 \mathrm{cP}$. The DDC weight was decreased from $18 \mathrm{~g}$ to $12 \mathrm{~g}$, while power consumption in the Tx or Rx mode and in the Drug Deployment mode were reduced from $33.8 \mathrm{~mA}$ to $24.2 \mathrm{~mA}$ and from $748 \mathrm{~mA}$ to $418 \mathrm{~mA}$, respectively.

Beyond the specific design, modeling, and assessment of the proposed coil-magnet-piston mechanism, the main contribution of this work is to illustrate how our component based design environment can be leveraged for the early validation of a novel MCR design. All the hardware and software components described in this paper are available open-source at pillforge.github.io and can be used as a template by the MCR research community.

Future work aims to expand the DDC template with modules for magnetic locomotion [3], anchoring for localized drug delivery, and magnetic localization [34] toward the end goal of intelligent drug delivery in the GI tract.

\section{Acknowledgment}

This material is based upon work supported in part by the Vanderbilt Institute in Surgery and Engineering and in part by the National Science Foundation under grants number CNS-1239355 and IIS-1453129. Any opinions, findings and conclusions or recommendations expressed in this material are those of the authors and do not necessarily reflect the views of the National Science Foundation.

\section{References}

[1] G. Iddan, G. Meron, A. Glukhovsky, S. P., Wireless capsule endoscopy, Nature 405 (6785) (2000) 417-418.

[2] S. P. R., , O. K. L., V. P., Technical research update in capsule endoscopy, World Journal of Gastroenterology 21 (2010) 1052810541.

[3] G. Ciuti, P. Valdastri, A. Menciassi, P. Dario, Robotic magnetic steering and locomotion of capsule endoscope for diagnostic and surgical endoluminal procedures, Robotica 28 (2010) 199-207.

[4] C. Quaglia, S. Tognarelli, E. Sinibaldi, N. Funaro, P. Dario, A. Menciassi, Wireless robotic capsule for releasing bioadhesive patches in the gastrointestinal tract, Journal of Medical Devices 8 (1) (2014) 014503.

[5] W. H. Crosby, U. Army, H. W. Kugler, Intraluminal biopsy of the small intestine, The American journal of digestive diseases 2 (5) (1957) 236-241.

[6] F. Munoz, G. Alici, W. Li, A review of drug delivery systems for capsule endoscopy, Advanced drug delivery reviews 71 (2014) 77-85.

[7] M. Beccani, H. Tunc, A. Taddese, E. Susilo, P. Volgyesi, A. Ledeczi, P. Valdastri, Systematic design of medical capsule robots, Design \& Test, IEEE 32 (5) (2015) 98-108.

[8] A. Taddese, M. Beccani, E. Susilo, P. Völgyesi, A. Lédeczi, P. Valdastri, Toward rapid prototyping of miniature capsule robots, in: Robotics and Automation (ICRA), 2015 IEEE International Conference on, ICRA 2015, 2015, in press.

[9] M. Beccani, E. Susilo, C. Di Natali, P. Valdastri, SMAC - a modular open source architecture for medical capsule robots, Int J Adv Robot Syst 11 (2014) 1-16.

[10] P. Valdastri, M. Simi, R. J. Webster III, Advanced technologies for gastrointestinal endoscopy, Annual Review of Biomedical Engineering 14 (5) (2012) 397-429.

[11] S. Stegemann, F. Leveiller, D. Franchi, H. De Jong, H. Lindén, When poor solubility becomes an issue: from early stage to proof of concept, European journal of pharmaceutical sciences 31 (5) (2007) 249-261. 
[12] J. F. Pinto, Site-specific drug delivery systems within the gastro-intestinal tract: from the mouth to the colon, International journal of pharmaceutics 395 (1) (2010) 44-52.

[13] P. J. van der Schaar, J. F. Dijksman, H. Broekhuizen-de Gast, J. Shimizu, N. van Lelyveld, H. Zou, V. Iordanov, C. Wanke, P. D. Siersema, A novel ingestible electronic drug delivery and monitoring device, Gastrointestinal endoscopy 78 (3) (2013) 520-528.

[14] D. M. Parkin, F. Bray, J. Ferlay, P. Pisani, Global cancer statistics, 2002, CA: a cancer journal for clinicians 55 (2) (2005) 74108.

[15] N. K. Shah, B. Rane, N. Gujarathi, Developments in colon specific drug delivery systems-a review, Pharma Science Monitor $5(2)$.

[16] A. Connor, Location, location, location: gastrointestinal delivery site and its impact on absorption., Therapeutic delivery 3 (5) (2012) 575

[17] D. Becker, J. Zhang, T. Heimbach, R. C. Penland, C. Wanke, J. Shimizu, K. Kulmatycki, Novel orally swallowable intellicap(R) device to quantify regional drug absorption in human gi tract using diltiazem as model drug, AAPS PharmSciTech 15 (6) (2014) 1490-1497.

[18] Scintipharma website: www.intuitivesurgical.com .

[19] I. Wilding, The enterion capsule: a novel technology for understanding the biopharmaceutical complexity of new molecular entities (nmes), Drug Deliv Tech 1 (1) (2001) 8-11.

[20] S. Yim, M. Sitti, Shape-programmable soft capsule robots for semi-implantable drug delivery, Robotics, IEEE Transactions on 28 (5) (2012) 1198-1202.

[21] S. H. Kim, K. Ishiyama, Magnetic robot and manipulation for active-locomotion with targeted drug release, IEEE/ASME Transactions on Mechatronics 19 (5) (2014) 1651-1659.

[22] S. P. Woods, T. G. Constandinou, Wireless capsule endoscope for targeted drug delivery: mechanics and design considerations, Biomedical Engineering, IEEE Transactions on 60 (4) (2013) 945-953.

[23] C. T. Dietzel, H. Richert, S. Abert, U. Merkel, M. Hippius, A. Stallmach, Magnetic active agent release system (maars): Evaluation of a new way for a reproducible, externally controlled drug release into the small intestine, Journal of Controlled Release 161 (3) (2012) 722-727.

24] W. Yu, R. Rahimi, M. Ochoa, R. Pinal, B. Ziaie, A smart capsule with gi-tract-location-specific payload release, Biomedical Engineering, IEEE Transactions on 62 (9) (2015) 2289-2295.

[25] H. Hafezi, T. L. Robertson, G. D. Moon, K.-Y. Au-Yeung, M. J. Zdeblick, G. M. Savage, An ingestible sensor for measuring medication adherence, Biomedical Engineering, IEEE Transactions on 62 (1) (2015) 99-109.

[26] C. Loudon, K. McCulloh, Application of the hagenpoiseuille equation to fluid feeding through short tubes, Annals of the Entomological Society of America 92 (1) (1999) 153-158.

[27] EN45502-1:2015, Implants for surgery. Active implantable medical devices. General requirements for safety, marking and for information to be provided by the manufacturer. (2015) 1-64.

[28] G. Karsai, J. Sztipanovits, A. Ledeczi, T. Bapty, Modelintegrated development of embedded software, Proceedings of the IEEE 91 (1) (2003) 145-164.

[29] M. Maróti, T. Kecskés, R. Kereskényi, B. Broll, P. Völgyesi, L. Jurácz, T. Levendoszky, Á. Lédeczi, Next generation (meta) modeling: Web-and cloud-based collaborative tool infrastructure, Proceedings of MPM (2014) 41

[30] P. Levis, S. Madden, J. Polastre, R. Szewczyk, K. Whitehouse, A. Woo, D. Gay, J. Hill, M. Welsh, E. Brewer, et al., Tinyos: An operating system for sensor networks, in: Ambient intelligence, Springer, 2005, pp. 115-148.

[31] P. Valdastri, A. Menciassi, P. Dario, Transmission power requirements for novel ZigBee implants in the gastrointestinal tract, Biomedical Engineering, IEEE Transactions on 55 (6) (2008) 1705-1710.

[32] Www.edn.com/design/pc-board/4429893/The-case-for-rigidflex-PCB-technology
[33] G. Tortora, P. Valdastri, E. Susilo, A. Menciassi, P. Dario, F. Rieber, M. O. Schurr, Propeller-based wireless device for active capsular endoscopy in the gastric district, Minimally invasive therapy \& allied technologies: MITAT : official journal of the Society for Minimally Invasive Therapy 18 (5) (2009) 280-90.

[34] C. Di Natali, M. Beccani, P. Valdastri, Real-time pose detection for magnetic medical devices, Magnetics, IEEE Transactions on 49 (7) (2013) 3524-3527. 